\title{
Resting State Brain Network Modeling Based On Functional Magnetic
}

\author{
Resonance Imaging \\ Ming Ke ${ }^{1, a^{*}}$,Zhijing $\mathrm{Li}^{1, \mathrm{~b}}$, Zhao Cao ${ }^{1, \mathrm{c}}$, Xiaoping Yang ${ }^{2, \mathrm{~d}}$ \\ ${ }^{1}$ College of Computer and Communication, Lanzhou University of Technology, Lanzhou, Gansu, \\ 730050, China \\ ${ }^{2}$ Department of Imaging Diagnosis, Lanzhou General Hospital of Lanzhou Military Command, \\ Lanzhou, Gansu, 730050, China.
}

akemingkitty@163.com, btokyojackson@126.com, caozhaozf@163.com, dlwyxp_zxl@sohu.com

Keywords: Complex network, Partial least squares, Pearson correlation, Functional magnetic resonance imaging

\begin{abstract}
In this paper, the functional magnetic resonance imaging (fMRI) technique and complex network method were used to study the brain functional network of normal subjects. We used the partial least squares (PLS) regression modeling method to construct the normal human brain function network. The global statistical properties of the brain network revealed the brain functional network had small-world effect. Through the evaluation of centrality indices, the gyri callosus, the supramarginal gyrus, gyri frontalis superior and the gyrus angularis were the key areas of the brain functional network in resting state. The result showed that compared with the Pearson correlation analysis method, the PLS algorithm was better to construct the brain network model. It is not only expressed in the brain network threshold is generally high, the "small world" attribute is more obvious, but also the key brain regions that were inferred are more accurate and more consistent with physiological results.
\end{abstract}

\section{Introduction}

The brain is the most complex and the most perfect information processing system currently. At present, the research of complex brain network[1-2] was a hot topic in the field of brain science research. As a kind of classical brain network modeling method, Pearson correlation analysis was widely used in the research of brain functional network, but there are also some shortcomings, such as it does not consider the number of data overlap. PLS was used to model the regression model under the condition of the existence of severe multiple correlation. Li et al. [3] used the PLS algorithm to carry out the function of the seven brain regions of normal human brain. The research showed that the PLS algorithm could effectively construct the brain functional network.

In this paper, the models of resting-state brain network were established based on complex network theory and method. Furthermore the normal human brain functional network properties were analyzed.

\section{Data acquisition and preprocessing}

All resting-state brain data were collected using a SIEMENS 3.0T nuclear magnetic resonance scanner. A total of 15 healthy volunteers participated in the experiment (Male 8 cases, female 7 cases). Scan parameters were as follows: repeat time $=2000 \mathrm{~ms}$, echo time $=30 \mathrm{~ms}$, Field of vision $=240 \mathrm{~mm}$, angle of rotation $=90^{\circ}$, matrix $=64 \times 64$, slice thickness $=3.8 \mathrm{~mm}$, gap $=0.38 \mathrm{~mm}$. A total of 200 time points were collected, and the scanning time was 400s. The whole data preprocessing procedure mainly includes format conversion, scan time correction, head motion correction, space 
standardization, smooth, remove the linear trend and low frequency filter.

\section{Brain Network Modeling}

Based on the data of fMRI experiment, the whole brain was divided into 90 brain regions according to the Anatomical Automatic Labeling (AAL).

PLS Regression Modeling. PLS algorithm[4] is a new method of multivariate statistical analysis, which is based on the method of component extraction. In the extraction of components, the prediction of the data information and the variable data information are considered, and make the maximum correlation between the data and the information from the forecast variables and the dependent variables. And then use the extracted components to establish the model. If the established model achieves the accuracy requirements, then terminate the operation of extraction, otherwise, the components are extracted from the remaining residual information, this process is repeated several times until reaching the modeling requirements.

Compared with the traditional multiple linear regression model, the characteristics of PLS regression was as follows: (1) It can be carried out under the condition of the existence of serious multiple correlation of the Independent variable. (2) It can be carried out under the condition of the number of sample points is less than the number of variables. (3) PLS regression model can more easily identify the system information and noise.

In order to avoid the edges of the network which were automatic connected, the diagonal elements are set to 0 . The setting of $T$ must satifies that the network average degree $\langle K>$ is not less than the natural logarithm of the number of nodes $(\ln (N))$, so as to conform to the actual characteristics of the human brain.

T Test Method. The statistical analysis of $a_{i j}$ in correlation coefficient $A$ of the PLS algorithm was carried out by using the single sample t test method[5], in order to detect the function of $i$ and $j$. Assume that: $H_{0}: \mu \leq T, H_{1}: \mu>T$. Among them: $\mu$ is the sanmple mean, $T$ is the correlation coefficient threshold for the specified. Use the $t=\frac{\bar{X}-T}{S / \sqrt{n}}$ for the test statistic. The rejection region of $H_{0}$ is as follows:

$$
t=\frac{\bar{X}-T}{S / \sqrt{n}} \geq t_{a}(n-1)
$$

When $\mathrm{t}$ falls in the rejection region, it shows that the intensity of the functional connection between $i$ and $j$ was significantly higher than that of $T$. The nodes $i$ and the node $j$ are connected by edges, otherwise it is not.

Network Attribute Analysis. We used the small world model[6] to study the characteristics of the functional network. When $\gamma=C_{\text {real }} / C_{\text {random }}>1, \lambda=L_{\text {real }} / L_{\text {random }} \approx 1$, it's considered that the network had the small world characteristics. Humphreys[7] and his companions used one indicator to measure the characteristics of "small world", when $\sigma>1$, the network has "small world" attribute, and the $\sigma$ is greater, the network's "small world" property is stronger. Among them, $C_{\text {real }} 、 C_{\text {random }}$ represent the clustering coefficient of the actual network and the random network, $L_{\text {real }} 、 L_{\text {random }}$ represent the shortest path length of the actual network and random network. However, the cluster coefficient only considers the direct connection between the neighbor nodes, and the shortest path length is usually performed in a connected graph. The study then used the Eloc (local efficiency) and the Eglob (global efficiency) to further measure the network properties of the brain network. The local efficiency of any node $i$ and the global efficiency were as follows, and among them, $G_{i}$ refers to the sub graph of the node's neighbors, and $l_{j k}$ refers the shortest path length between node $i$ and node $j$.

$$
E(i)=\frac{1}{N_{G_{i}}\left(N_{G_{i}}-1\right)} \sum_{j \neq k \in G_{i}} \frac{1}{l_{j, k}}
$$




$$
E_{\text {glob }}=\frac{1}{N(N-1)} \sum_{i, j \in V, i \neq j} \frac{1}{l_{i j}}
$$

\section{Results and Discussion}

Figure 1 and Figure 2 showed the average degree of the network under the different threshold of the Pearson algorithm and PLS. From Figure 1, we could see that the choice of the threshold $T=0.3$ was appropriate, from the figure 2, we could see that the choice of the threshold $T=0.4$ was appropriate. At this point, the average degree of the network was greater than the number of network nodes $N$ 's natural logarithm.

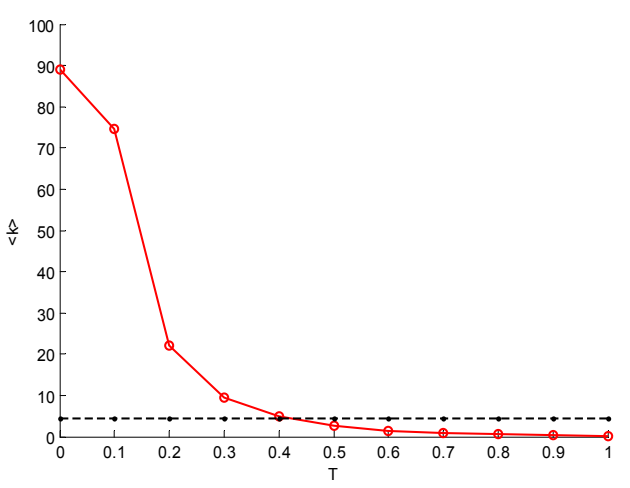

Fig 1The average degree of the network under different thresholds of Pearson algorithm

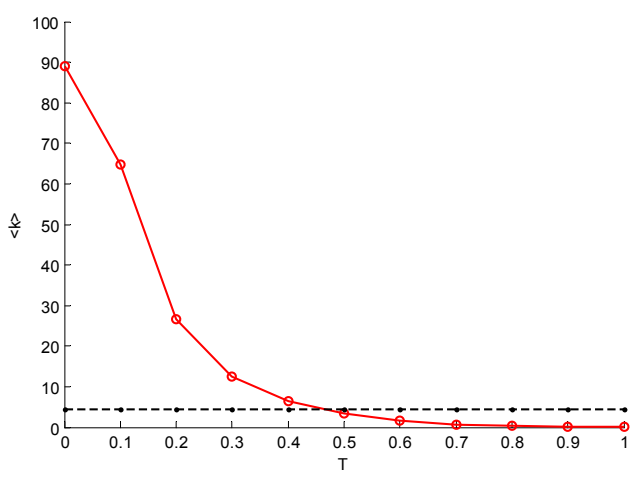

Fig 2The average degree of the network under different thresholds of the PLS

Tab 1 parameters' results of Pearson algorithm and PLS

\begin{tabular}{|c|c|c|c|c|c|c|c|}
\hline & $\mathrm{C}$ & $\mathrm{L}$ & $\gamma$ & $\lambda$ & $\sigma$ & Eloc & Eglob \\
\hline Pearson & 0.4806 & 2.5573 & 4.6979 & 1.2616 & 3.5415 & 1.7969 & 0.4009 \\
PLS & 0.2956 & 1.0447 & 4.4776 & 0.414 & 10.8155 & 10.6916 & 0.1752 \\
Random & 0.1023 & 2.027 & & & & & \\
\hline
\end{tabular}

Note: Random represents random network; $C$ represents cluster coefficient; L represents the shortest path length; $\gamma=C_{\text {real }} / C_{\text {random }} ; \lambda=L_{\text {real }} L_{\text {random }} ; \sigma=\gamma / \lambda$.

From table 1 it was seen that $\sigma=3.542$ and 10.8155 , they were greater than 1 , it meaned that the normal human resting-state brain functional network constructed by Pearson algorithm and PLS had the properties of "small world". Contrast Figure 1 and Figure 2 it was seen that under the same threshold, the average degree of brain network constructed by the PLS algorithm was significantly higher than that of by the Pearson correlation analysis method. Furthermore, the Eloc of PLS was 10.691, and the $\sigma$ of PLS was 10.815, which were significantly larger than the value of Pearson correlation analysis method. It illustrated that the PLS algorithm could take into account more efficient connections, including direct and indirect connections.

We measured the degree of network centrality to find out the key resting-state brain regions. The central index included the betweenness[8] of nodes and the degree of the nodes. All nodes in the network were sorted from high to low, found the top 10 nodes of the highest degree, and correspond them to specific locations in the brain, the results were shown in Table 2 and table 3 . From table 2, Insula-L, Insula-R, and SupraMarginal-R had been the high degree and betweenness. From table 3 Frontal_Sup_Orb_L, Cingulum_Post_L, Angular_L, SupraMarginal_L, Frontal_Sup_Medial_L had been the high degree and betweenness. It indicated that these brain regions were connected to other regions widely, and so they are the key brain regions. In relative terms, the partial least squares algorithm could predict more key brain regions that are more accurate and more consistent with physiological results. 
Tab 2 Brain areas corresponding to first 10 nodes with highest value of degree and betweenness centrality in the Pearson algorithm

\begin{tabular}{|c|c|}
\hline degree & betweenness \\
\hline AAL brain region & AAL brain region \\
\hline Insula_L & Insula_R \\
Insula_R & SupraMarginal_R \\
SupraMarginal_R & Frontal_Sup_Orb_L \\
Cingulum_Post_L & Lingual_R \\
Rolandic_Oper_R & Frontal_Inf_Oper_R \\
Angular_L & Insula_L \\
Rolandic_Oper_L & Calcarine_L \\
Frontal_Sup_Medial_R & Frontal_Mid_Orb_R \\
Frontal_Sup_Medial_L & Cingulum_Post_L \\
SupraMarginal_L & Olfactory_R \\
\hline
\end{tabular}

Tab 3 Brain areas corresponding to first 10 nodes with highest value of degree and betweenness centrality in the PLS

\begin{tabular}{|c|c|}
\hline degree & betweenness \\
\hline AAL brain region & AAL brain region \\
\hline Angular_L & Frontal_Sup_Orb_L \\
Cingulum_Post_L & Cingulum_Post_L \\
Frontal_Sup_Orb_L & Insula_R \\
Angular_R & Angular_L \\
Parietal_Inf_R & Frontal_Mid_Orb_R \\
SupraMarginal_R & SupraMarginal_R \\
Parietal_Sup_R & Temporal_Pole_Sup_L \\
Cingulum_Post_R & Lingual_L \\
Frontal_Sup_Medial_- & Frontal_Mid_Orb_L \\
LRolandic_Oper_R & Frontal_Sup_Medial_L \\
\hline
\end{tabular}

\section{Conclusion}

In this study, we used PLS regression modeling to analyze resting-state functional magnetic resonance data and establish the models of brain functional network. We also analyzed the functional connectivity of brain network, and it was found that the brain functional network has the characteristics of "small world". The results show that the PLS algorithm was effectively constructed the brain functional network, and the PLS was better method than Pearson correlation method in modelling the brain functional network.

\section{Acknowledgment}

The work was partially supported by the National Science Foundation of China (61263047), and the Fundamental Research Funds for Gansu Universities.

\section{Reference}

[1]X. Qu. Construction and analysis of complex brain networks based on time series. Electronic technology and software engineering, 2015,5:19.

[2]X. Wang, Xiang Li, Guanrong Chen. Complex network theory and application. Beijing: Tsinghua University press, 2006.

[3]Y. Li, R. Shen, H. Dai, W. Li. Exploration of the brain network connection modes Based on resting state fMRI data. Chinese Journal of Medical Physics, 2013,30:3898-3902.

[4]P. Wang. The application of partial least squares regression and kernel partial least squares regression. Kunming University of Science and Technology, 2014:17-28.

[5]M. Ke. Research and application of brain functional network model based on functional magnetic resonance imaging. Lanzhou University of Technology, 2009:44-46.

[6] D.J. Watts, S.H. Strogatz. Collective dynamics of 'small-world' networks. Nature, 1998, 393(6684): 440-442.

[7]M.D. Humphries,K. Gurney,T.J. Prescott. The brainstem reticular formation is a small world, not scale-free, network. Proc R Soc Lond BBiol Sci,2006,273:503-511.

[8]H. Huo. Research on average betweenness in the network. Qinghai Normal University, 2014:9-13. 